\title{
Precise Application of the Xiao-Chai-Hu-Tang in 98 Cases of Patients with the Major Syndrome of Feeling Pain and Tenderness under the Right Costal Arch
}

\author{
Wang Zhi-qiang ${ }^{1}$, Song Zhi-yong ${ }^{2}$, Yang Bai-zhi ${ }^{1}$, Wang Xiu-hong ${ }^{3}$, Gui Huan-chen ${ }^{4}$, Gao Zhao-wang 5 \\ ${ }^{1}$ Department of Urology, Shouguang Hospital of Traditional Chinese Medicine(SHTCM), Shouguang, P.R.China \\ Wang Zhi-qiang, TCMwangzhiqiang@163.com, https://orcid.org/0000-0003-1459-6602. \\ Yang Baizhi, https://orcid.org/0000-0003-3444-4497. \\ ${ }^{2}$ Department of Anesthesiology, SHTCM,https://orcid.org/0000-0003-1940-7217 \\ ${ }^{3}$ Department of Outpatient, SHTCM , https://orcid.org/0000-0003-1130-6104 \\ ${ }^{4}$ Department of Pulmonary Medicine, SHTCM, https://orcid.org/0000-0003-1276-5468 \\ ${ }^{5}$ Department of Urology, Shandong University of Traditional Chinese Medicine Affiliated Hospital, Jinan, P.R.China, \\ https://orcid.org/0000-0002-7337-1457
}

Received:1 December 2019 Accepted: 5 December 2019 Published: 31 December 2019

Cite this Article Wang Zhi-qiang, Song Zhi-yong, Yang Bai-zhi, Wang Xiu-hong, Gui Huan-chen, Gao Zhao-wang: Precise Application of the Xiao-Chai-Hu-Tang in 98 Cases of Patients with the Major Syndrome of Feeling Pain and Tenderness under the Right Costal Arch [J].Medical Research,2019.1(1):26-30, http://dx.doi.org/10.6913/M RHK.201912_1(1).0005

Copyright (C) 2020 Creative Publishing Co., Limited. All rights reserved. Email:kycbshk@gmail.com.

\begin{abstract}
Xiao-Chai-Hu-Tang (XCHT), a representative of previous edition of Formulas of Traditional Chinese Medicine and harmonizing formulas, has multiple pharmacological functions. However, a variety of side effects could be caused by misuse or abuse, ignoring the theory of diagnosis and treatment based on the combination of syndrome and disease differentiation. Therefore, we conducted a retrospective study to evaluate the efficacy of XCHT, depending upon the individual patient's condition. Among 98 patients treated with XCHT, most of the patients of syndromes could be cured after about two course. The therapeutic effect rate of prescribed XCHT in our 98 cases of treatment is $91.84 \%$ (90/98). In addition, the high frequently syndromes are consistent with Shanhanlun.

With multiple years of experience, we believe that: patient feel pain and tenderness under the right costal arch is one of the symptoms of patients who fit for the treatment with XCHT.
\end{abstract}

Key words: diagnosis; Xiao-Chai-Hu-Tang (XCHT); syndrome; therapeutic effect

Xiao-Chai-Hu-Tang (XCHT), the famous classical prescription, was earliest reported in the ancient Chinese medical book Shanghanlun over 2000 years ago $^{[1]}$. It consists of Bupleurum falcatum, Panax ginseng, Scutellaria baicalensis, Pinellia ternate, Glycyrrhiza glabra, Zingiber officinale, and Zizyphus jujube $^{[2,3]}$. With these 7 simple types of medicinal herbs, XCHT combines the therapy of cooling with warming and is homological in ascending and descending and works on clearing and tonifying ${ }^{[4]}$. As the representative of previous edition of Formulas of Traditional Chinese Medicine and harmonizing formulas, XCHT takes "mediation" as a functional feature, which can be used in exogenous febrile diseases and miscellaneous diseases of internal injury. Generations of physicians extend and derive its connotation based on "mediation". The common saying, "Xiao-Chai-Hu-Tang cure for all disease", represents XCHT's wide range of applications and its impressive curative effects. However, a variety of side effects could be caused by misuse or abuse, ignoring the theory of diagnosis and treatment based on the combination of syndrome 
Medical Research ISSN 2664-0333 Volume 1 No.1 PP.26-30 http://dx.doi.org/10.6913/MRHK.201912_1(1).0005 Wang Zhi-qiang,et al: Precise Application of the Xiao-Chai-Hu-Tang in 98 Cases of Patients with the Major Syndrome of Feeling Pain and Tenderness under the Right Costal Arch and disease differentiation. Therefore, we conducted a retrospective study to evaluate the efficacy of XCHT, depending upon the individual patient's condition. We summarize it as below:

\section{CLINICAL DATA}

In this retrospective study, we reviewed the data of 100 patients (56 male and 44 female, range 3-78 years) who had prescribed Xiao-Chai-Hu-tang from June 2009 to May 2017 at Shouguang Hospital of Traditional Chinese Medicine and Shandong University of Traditional Chinese Medicine Affiliated Hospital.

\section{DIAGNOSTIC CRITERIA}

The diagnostic criteria are met with the major syndrome and the one of secondary syndromes. The major syndrome is that the patient feel pain and tenderness under the right costal arch. The secondary syndrome were as follows: (1) intermittent chills and fevers, (2) a feeling of distention and oppression in the chest and costal region, (3) anorexia and distinclination to talk, (4) restless and nauseous, (5) bitterness in the mouth, (6) a parched throat, (7) vertigo, (8) pulse string.

\section{TREATMENTS}

The treatment was in accordance with the modification of XCHT in Shanghanlun. To be restless but not nauseous: Take away Pinellia ternate and Panax ginseng, and add Fructus Trichosanthis; With thirst for water: Take away Pinellia ternate, increase Panax ginseng to four and a half liang, add Radix Trichosanthis; With abdominal pain: Take away Scutellaria baicalensis, add Radix Paeoniae; With mass below the costal margin: Take away Zizyphus jujube, and add Concha Ostreae; With palpitation and dysuria: Take away Scutellaria baicalensis, and add Poria; No thirst of water but with a slight exterior fever: Take away Panax ginseng, and add Ramulus Cinnamomi. Cover the patient with a quilt to obtain a light perspiration; Accompanied by coughing: Take away Panax ginseng, Zingiber officinale and Zizyphus jujube; add Fructus Schisandrae and Rhizoma Zingiberis. Five days count as a course, and the total treatment time is two courses or until the patient is cured.

\section{THERAPEUTIC CRITERIA}

Cured: major syndrome and secondary syndromes disappeared; Effective: major syndrome significantly decreased and secondary syndromes were significantly alleviated; Ineffective: the symptoms were not significantly improved.

\section{RESULTS}

With the exception of one patient who was unable to fulfill timely follow-up and another patient who was transferred to another hospital due to aggravation in the primary diseases, the therapeutic effect for the other 98 patients is shown in Table 1. Among these patients, most of the patients of syndromes could be cured after about two course. The high frequently syndromes are consistent with Shanhanlun (Table 2).

Table 1. Therapeutic Effect of Prescribed XCHT

\begin{tabular}{|c|c|c|c|c|}
\hline Patients & Cured & Effective & Ineffective & $\begin{array}{c}\text { Total Effective } \\
\text { Rate }(\%)\end{array}$ \\
\hline 98 & 84 & 6 & 8 & 91.84 \\
\hline \multicolumn{5}{|c|}{ Table 2. The High Frequency Syndromes of XCHT } \\
\hline \multicolumn{3}{|c|}{ Syndrome } & Frequency & Ratio (\%) \\
\hline \multicolumn{3}{|c|}{ Bitterness in the mouth } & 45 & 45.92 \\
\hline \multicolumn{3}{|c|}{ Reluctant to speak and eat } & 45 & 45.92 \\
\hline \multicolumn{3}{|c|}{ Nauseous } & 41 & 41.84 \\
\hline \multicolumn{3}{|c|}{ Intermittent chills and fevers } & 27 & 27.55 \\
\hline \multicolumn{3}{|c|}{ Parched throat } & 26 & 26.53 \\
\hline
\end{tabular}


Medical Research ISSN 2664-0333 Volume 1 No.1 PP.26-30 http://dx.doi.org/10.6913/MRHK.201912_1(1).0005 Wang Zhi-qiang,et al: Precise Application of the Xiao-Chai-Hu-Tang in 98 Cases of Patients with the Major Syndrome of Feeling Pain and Tenderness under the Right Costal Arch
Vertigo
25
25.51

Feels a distention and a sensation of oppression in the chest and

$$
\text { costal region }
$$

$21 \quad 21.43$

Restless

20

20.41

Headache

17

17.35

\section{DISCUSSION}

Xiao-Chai-Hu-Tang also called Minor Bupleurum Decoction is traditionally used in Japan, Korea and China ${ }^{[5]}$. The preparation of formulas in which herbs are combined to achieve greater efficacy than individual herb is a form of oriental herbology. These herbal formulas are commonly used for treatment of a number of disorders ${ }^{[6]}$. Previous studies demonstrated that XCHT has multiple pharmacological functions, such as

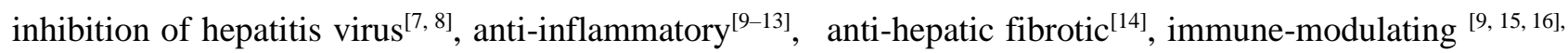
antioxidant $^{[17]}$, anti-cancer ${ }^{[18-20]}$, liver protective effect ${ }^{[21-23]}$, and renal protective effect ${ }^{[24-26]}$. However, Chinese herbal medicine should be specifically used depending upon a syndrome, not a disease, and a number of side effects could be caused by the misuse or abuse without any consideration of syndrome differentiation ${ }^{[27]}$. Reports have raised concerns regarding acute respiratory failure and interstitial pneumonia ${ }^{[28]}$. Indeed, the patients who took XCHT for treating chronic liver diseases have cases of interstitial pneumonia ${ }^{[5]}$. According to Lee ${ }^{[29]}$ et al, the incidence of side effects could be increased by coadministration of interferon, the duration of XCHT treatment and increasing age of patients. Therefore, it is very difficult to tell whether or not the apparent side effects are causally linked to XCHT administration.

With multiple years of experience, we believe that: patient feel pain and tenderness under the right costal arch is one of the symptoms of "When one of the symptoms of syndrome is observed, a diagnosis of Xiao-Chai-Hu tang syndrome can be established." Other examples include Triple-jiao and Gallbladder, which are associated with Couli and vellus hair (Cou is a juncture where the Triple-jiao and Body Resistance converge and a Channel where in vital energy and the blood flow. Li is the texture on skin, viscera and bowels.) ${ }^{[30]}$. When the blood is deficient and Vital Resistance weaken, the Couli opens, so the pathogenic factor intrudes into Couli through the vellus hair and enters the triple-jiao channel of hand shaoyang; then the triple-jiao channel of hand shaoyang intersect with the gallbladder channel of foot shaoyang on the neck and shoulder. Consequently, pathogenic factors enter the gallbladder Channel of Foot-Shaoyang; Then through quepen (supraclavicular fossa), gallbladder channel connect to the liver and the gallbladder attaches to the liver, the pathogenic factors enter the gallbladder. On the other hand, liver is located at the left side and lungs are located at the right side, spirit and essence circulation refer to left as ascending and right as descending ${ }^{[31]}$. The lung governs qi, externally it connects with skin and body fair. The pathogenic factors pass through upper-jiao with lung qi fall from the right side, then stagnate in the subcostal; consequently, the patient feels pain and tenderness under the right costal arch. The evidence exhibited is caused by the pathogenic factors' struggles against the body resistance at the costal region. The syndromes include feeling of distention and oppression in the chest and costal region, anorexia and distinclination to talk, restless and nauseous.

In summary, among 98 patients treated with XCHT, most of the patients of syndromes could be cured after about two course. The therapeutic effect rate of prescribed XCHT in our 98 cases of treatment is $91.84 \%$ (90/98). In addition, the high frequently syndromes are consistent with Shanhanlun. With multiple years of experience, we believe that: patient feel pain and tenderness under the right costal arch is one of the symptoms of patients who fit for the treatment with XCHT.

\section{REFERENCES}

1. Qin XK, Li P, Han M, et al. Xiaochaihu Tang for treatment of chronic hepatitis B: a systematic review of randomized trials. Zhong Xi Yi Jie He Xue Bao. 2010; 8:312-320. 
Medical Research ISSN 2664-0333 Volume 1 No.1 PP.26-30 http://dx.doi.org/10.6913/MRHK.201912_1(1).0005 Wang Zhi-qiang,et al: Precise Application of the Xiao-Chai-Hu-Tang in 98 Cases of Patients with the Major Syndrome of Feeling Pain and Tenderness under the Right Costal Arch

2. Fujiwara K, Mochida S, Nagoshi S, et al. Regulation of hepatic macrophage function by oral administration of xiao-chai-hu-tang (sho-saiko-to, TJ-9) in rats. J Ethnopharmacol. 1995 May;46(2):107-14.

3. Zheng NN, Dai JY, Cao HJ, et al. Current Understanding on Antihepatocarcinoma Effects of Xiao Chai Hu Tang and Its Constituents. Evid Based Complement Alternat Med. 2013; 2013:529458.

4. Li N, Gao F, Wang XY. Application of Xiaochaihu Decoction combined with case analysis. China Journal of Traditional Chinese Medicine and Pharmacy. 2013; 28(6):1784-6.

5. Kwang HS, Yun HK, Bu-Yeo K, et al. Sho-saiko-to, a traditional herbal medicine, regulates gene expression and biological function by way of microRNAs in primary mouse hepatocytes. BMC Complement Altern Med. 2014; $14: 14$.

6. In SS, Mee YL, Yongbum K, et al. Subacute toxicity and stability of Soshiho-tang, a traditional herbal formula, in Sprague-Dawley rats. BMC Complement Altern Med. 2012; 12: 266.

7. Chang JS, Wang KC, Liu HW, et al. Sho-saiko-to (Xiao-Chai-Hu-Tang) and crude saikosaponins inhibit hepatitis B virus in a stable HBV-producing cell line. Am J Chin Med. 2007;35(2):341-51.

8. Cheng PW, Ng LT, Lin CC. Xiao chai hu tang inhibits CVB1 virus infection of CCFS-1 cells through the induction of Type I interferon expression. Int Immunopharmacol. 2006 Jun;6(6):1003-12.

9. Horie Y, Kajihara M, Yamagishi Y, et al. A Japanese herbal medicine, Sho-saiko-to, prevents gut ischemia/reperfusion-induced hepatic microvascular dysfunction in rats. J Gastroenterol Hepatol. 2001 Nov;16(11):1260-6.

10. Miyahara M, Tatsumi Y. Suppression of lipid peroxidation by sho-saiko-to and its components in rat liver subcellular membranes. Yakugaku Zasshi. 1990 Jun;110(6):407-13.

11. Yoshida K, Mizukawa H, Honmura A, et al. The effect of sho-saiko-to on the concentration of acid soluble glycoprotein in serum and on granuloma formation in carrageenin cotton pellet-induced granuloma rats. Am $\mathrm{J}$ Chin Med. 1993;21(2):171-7.

12. Yoshida K, Mizukawa H, Honmura A, et al. The effect of sho-saiko-to on concentration of vitamin E in serum and on granuloma formation in carrageenin cotton pellet-induced granuloma rats. Am J Chin Med. 1994;22(2):183-9.

13. Kusunose M, Qiu B, Cui T, et al. Effect of Sho-saiko-to extract on hepatic inflammation and fibrosis in dimethylnitrosamine induced liver injury rats. Biol Pharm Bull. 2002 Nov;25(11):1417-21.

14. Chen MH, Chen JC, Tsai CC, et al. Sho-saiko-to prevents liver fibrosis induced by bile duct ligation in rats. Am J Chin Med. 2004;32(2):195-207.

15. Deng G, Kurtz RC, Vickers A, et al. A single arm phase II study of a Far-Eastern traditional herbal formulation (sho-sai-ko-to or xiao-chai-hu-tang) in chronic hepatitis C patients. J Ethnopharmacol. 2011 Jun 14;136(1):83-7.

16. Chen MH, Chen JC, Tsai CC, et al. The role of TGF-beta 1 and cytokines in the modulation of liver fibrosis by Sho-saiko-to in rat's bile duct ligated model. J Ethnopharmacol. 2005 Feb 10;97(1):7-13.

17. Shiota G, Maeta Y, Mukoyama T, et al. Effects of Sho-Saiko-to on hepatocarcinogenesis and 8-hydroxy-2'-deoxyguanosine formation. Hepatology. 2002 May;35(5):1125-33.

18. Huang Y, Marumo K, Murai M. Antitumor effects and pharmacological interaction of xiao-chai-hu-tang (sho-saiko-to) and interleukin 2 in murine renal cell carcinoma. Keio J Med. 1997 Sep;46(3):132-7.

19. Ito H, Shimura K. Effects of a blended Chinese medicine, xiao-chai-hu-tang, on Lewis lung carcinoma growth and inhibition of lung metastasis, with special reference to macrophage activation. Jpn J Pharmacol. 1986 Jul;41(3):307-14.

20. Zhu K, Fukasawa I, Furuno M, et al. Inhibitory effects of herbal drugs on the growth of human ovarian cancer cell lines through the induction of apoptosis. Gynecol Oncol. 2005 May;97(2):405-9.

21. Itoh $\mathrm{S}$, Marutani $\mathrm{K}$, Nishijima $\mathrm{T}$, et al. Liver injuries induced by herbal medicine, syo-saiko-to (xiao-chai-hu-tang). Dig Dis Sci. 1995 Aug;40(8):1845-8. 
Medical Research ISSN 2664-0333 Volume 1 No.1 PP.26-30 http://dx.doi.org/10.6913/MRHK.201912_1(1).0005 Wang Zhi-qiang,et al: Precise Application of the Xiao-Chai-Hu-Tang in 98 Cases of Patients with the Major Syndrome of Feeling Pain and Tenderness under the Right Costal Arch

22. Amagaya S, Hayakawa M, Ogihara Y, et al. Treatment of chronic liver injury in mice by oral administration of xiao-chai-hu-tang. J Ethnopharmacol. 1989 Apr;25(2):181-7.

23. Zheng NN, Dai JY, Cao HJ. Current Understanding on Antihepatocarcinoma Effects of Xiao Chai Hu Tang and Its Constituents. Evid Based Complement Alternat Med. 2013; 2013: 529458.

24. Lin CC, Lin LT, Yen MH, et al. Renal protective effect of xiao-chai-hu-tang on diabetic nephropathy of type 1-diabetic mice. Evid Based Complement Alternat Med. 2012;2012:984024.

25. Rongjin Sun, Min Zeng, Ting Du, et al. Simultaneous Determinations of 17 marker compoundsin Xiao-Chai-Hu-Tang by LC-MS/MS: Application to its Pharmacokinetic Studies in Mice. J Chromatogr B Analyt Technol Biomed Life Sci. 2015 Oct 15; 1003: 12-21.

26. Lin CC, Lin LT, Yen MH. Renal Protective Effect of Xiao-Chai-Hu-Tang on Diabetic Nephropathy of Type 1-Diabetic Mice. Evid Based Complement Alternat Med. 2012; 2012: 984024.

27. Wang ZQ, Yuan L, Wang YC, et al. Efficacy and safety of Chinese herbal medicine for chronic prostatitis associated with damp-heat and blood-stasis syndromes: a meta-analysis and literature review. Patient Prefer Adherence. 2016; 10: 1889-1902.

28. Chang JS, Wang KC, Liu HW, et al. Sho-saiko-to (Xiao-Chai-Hu-Tang) and crude saikosaponins inhibit hepatitis B virus in a stable HBV-producing cell line. Am J Chin Med. 2007;35(2):341-51.

29. Lee MY, Seo CS, Shin IS, et al. Evaluation of Oral Subchronic Toxicity of Soshiho-Tang Water Extract: The Traditional Herbal Formula in Rats. Evid Based Complement Alternat Med. 2013; 2013: 590181.

30. Huang YX. Inspiration on Sweat inducing Method by'striae and interstitial space and vellus hair corresponding to Sanjiao and gallbladder’. World Chinese Medicine. 2012; 7(1): 9-10.

31. Li HJ, Liu XG. Observations on the therapeutic effiect of acupuncture with left ascending and right descending method for 52 cases of chronic diarrhea. World Journal of Acupuncture-Moxibustion, 2013, 23(4), 36-39. 